stillbirth, fetal loss, neonatal death, low birth weight, preterm birth and syphilis infection in the infant. In Haiti, 90\% of pregnant women report at least one antenatal visit. We evaluated the field performance of the SD BIOLINE HIV/Syphilis Duo test in a high-risk setting in Port-au-Prince, Haiti using whole blood fingerprick specimens.

Methods GHESKIO (Haitian Study Group for Kaposi's sarcoma and Opportunistic Infections) clinic attendees 18 years of age or older were invited to participate. Venipuncture blood specimens were used for reference testing: for HIV, Murex HIV-1.2.0 (DiaSorin S.p. A.) or Determine HIV-1/2 (Alere Inc). Positive results were confirmed with the HIV $(1+2)$ Rapid Test Strip (KHB Shanghai Kehua Bioengineering Co. Ltd). For Treponema pallidum (Tp) antibody comparison, Treponema Pallidum Hemagglutination Assay (TPHA) (Human Gesellschaft fur Biochemica und Diagnostica $\mathrm{mbH}$ ) was used. For 21 TPHA indeterminate results, specimens were retested using a Tp enzyme-linked immunosorbent assay test (ELISA) (Architect Syphilis Tp, Abbott Laboratories). Sensitivity and specificity were calculated and the exact binomial method was used to determine $95 \%$ confidence intervals (CI).

Results Of 298 study participants, 61 (20.5\%) were male. Of 237 females, 49 (20.7\%) were pregnant. For the HIV component, sensitivity and specificity were 99.2\% (95\% CI: 95.8\%, 100\%) and 97.0\% (95\% CI: 93.2\%, 99.0\%), respectively. All 21 TPHA indeterminate results were Tp ELISA reactive. For the Tp component, sensitivity and specificity were 96.5\% (95\% CI: 91.2\%, 99.0\%) and 90.8\% (95\% CI: 85.7\%, 94.6\%), respectively. In pregnant women, the HIV component sensitivity and specificity were $93.3 \%$ (95\% CI: 68.0\%, 99.8\%) and 94.1\% (95\% CI: $80.3 \%, 99.3 \%)$, respectively; and for the Tp component were 100\% (95\% CI: $81.5 \%, 100 \%)$ and $96.8 \%(83.3 \%$, 99.9\%), respectively.

Conclusion The HIV antibody component of the Duo test shows excellent performance. The Treponema pallidum antibody component showed high sensitivity, and slightly lower specificity. Amongst pregnant women the test performed very well.

Disclosure of interest statement The study was supported in part by Standard Diagnostics.

\section{P07.21 SERUM CYTOKINE ANALYSIS AMONG PATIENTS WITH AND WITHOUT EARLY SYPHILIS INFECTION}

${ }^{1} \mathrm{CC}$ Bristow*, ${ }^{2} \mathrm{H}$ Maecker, ${ }^{2} \mathrm{Y}$ Rosenberg-Hasson, ${ }^{3} \mathrm{SR}$ Leon, ${ }^{3} \mathrm{SK}$ Vargas, ${ }^{1} \mathrm{KA}$ Konda, ${ }^{3} \mathrm{CF}$ Caceres, ${ }^{1} \mathrm{JD}$ Klausner. 'University of California Los Angeles; ${ }^{2}$ Human Immune Monitoring Center, Institute for Immunity, Transplantation, and Infection, Stanford University School of Medicine, Stanford, CA, USA; ${ }^{3}$ Laboratory of Sexual Health and Unit of Health, Sexuality and Human Development, Universidad Peruana Cayetano Heredia

\subsection{6/sextrans-2015-052270.337}

Introduction Current diagnostic technology for syphilis is over 100 years old and relies on detection of either reagins or treponemal antibodies. The development of new tests formulated on the basis of host cellular response may allow for improved diagnosis and clinical management. We aimed to pilot an evaluation of sera cytokine profiles as a means to better understand the pathogenesis of early infections.

Methods Participants included men who have sex with men and transgender women with and without early syphilis recruited at two sexual health clinics in Lima, Peru. Median fluorescence intensity (MFI) of 63 cytokines in serum collected on day of diagnosis was measured using Luminex (eBioscience). We calculated the relative change in MFI between those with active (RPR titer $\geq 1: 32$, TPPA + ) versus no syphilis infection (TPPA-) and compared groups using a two-sample t-test.

Results Among 5 participants with active syphilis infections and 5 without there were 11 cytokines that differed between the groups with a p-value $<0.1$. The relative change (ratio) in MFI between syphilis infected and syphilis uninfected specimens was 1.535 for interleukin-7 ( $\mathrm{p}=0.012$ ), 2.062 for inducible protein$10(\mathrm{p}=0.054), 2.390$ for leptin $(\mathrm{p}=0.067), 1.960$ for vascular endothelial growth factor $(\mathrm{p}=0.067), 1.712$ for granulocytemacrophage colony-stimulating factor $(\mathrm{p}=0.069), 1.947$ for interleukin-10 ( $\mathrm{p}=0.070), 3.479$ for vascular endothelial growth factor D ( $\mathrm{p}=0.074), 0.687$ for Eotaxin $(\mathrm{p}=0.082)$, 2.532 for macrophage inflammatory protein-1beta $(p=0.090)$, 1.493for monocyte chemotactic protein-3 ( $\mathrm{p}=0.093)$, and 2.526 for platelet-derived growth factor-BB $(\mathrm{p}=0.097)$.

Conclusion Cytokines associated with cellular immune response might be useful in differentiating those needing treatment for active syphilis from those not requiring treatment. All of the cytokines presented here are higher in the active syphilis group versus syphilis uninfected except for Eotaxin. Larger sample size and longitudinal data are required to characterise cytokine profiles associated with treatment response and cure. Identifying cytokine changes may provide a new opportunity for diagnostic testing for syphilis infection.

Disclosure of interest statement None.

\section{P07.22 PERFORMANCE CHARACTERISTICS OF AN AUTOMATED ASSAY ON THE COBAS ${ }^{\circledR} 4800$ SYSTEM TO DETECT HERPES SIMPLEX VIRUS FROM GENITAL LESION SPECIMENS WITH THE COBAS ${ }^{\circledR}$ HSV 1 AND 2 TEST}

${ }^{1} \mathrm{M}$ Chio, ${ }^{1} S$ Aminah, ${ }^{2} \mathrm{~J}$ Osiecki ${ }^{*},{ }^{2} \mathrm{M}$ Lewinski, 'L Low. 'Department of Sexually Transmitted Infections Control Clinic, Singapore; ${ }^{2}$ Roche Molecular Diagnostics, Pleasanton, CA, USA

\subsection{6/sextrans-2015-052270.338}

Introduction Herpes simplex viruses occur worldwide and laboratory confirmation is recommended using methods that directly demonstrate presence of the virus in genital specimens. This study was conducted to establish performance characteristics for the cobas ${ }^{\circledR}$ HSV 1 and 2 Test by evaluating genital lesion samples compared to standard of care viral isolation.

Methods This evaluation study was conducted using 193 clinical swab specimens from active lesions of suspected herpes simplex collected at the DSC Clinic during the period of April 2014 through October 2014. The results of the cobas ${ }^{\circledR}$ HSV 1 and 2 Test were compared with those obtained from viral isolation. Samples with discordant results for HSV-1 and HSV-2 were confirmed with an in-house HSV PCR performed at Singapore General Hospital.

Results Of the 193 samples tested for HSV-1, 27 were positive and 159 were negative with both the cobas ${ }^{\circledR}$ HSV 1 and 2 Test and viral isolation. Of the samples evaluated, 7 were positive by the cobas ${ }^{\circledR}$ HSV 1 and 2 Test but not in viral isolation. The overall agreement of the cobas ${ }^{\circledR}$ HSV 1 and 2 Test and viral isolation for HSV-1 was 96.4\%. Of the 193 samples tested for HSV-2, 57 were positive and 113 were negative with both the cobas ${ }^{\circledR}$ HSV 1 and 2 Test and viral isolation. There were 23 samples found to be positive with the cobas ${ }^{\circledR}$ HSV 1 and 2 Test but not in viral isolation. The overall agreement of the cobas ${ }^{\circledR}$ HSV 1 and 2 Test and viral isolation for HSV-2 was 88.1\%. Further testing of discrepant samples revealed 20/23 were detected by an in-house HSV PCR assay. 
Conclusion The cobas ${ }^{\circledR}$ HSV 1 and 2 Test, run on the fully automated cobas ${ }^{\circledR} 4800$ system, demonstrated excellent performance for detecting HSV 1 and 2 from clinical specimens when compared with viral isolation.

Disclosure of interest statement The Department of Sexually Transmitted Infections Control Clinic, Singapore collaborated with Roche Molecular Systems on the presentation of the outcomes of this evaluation study.

\section{P07.23 EVALUATION OF THE COBAS ${ }^{\circledR}$ HSV 1 AND 2 TEST FOR THE DETECTION OF HSV FROM CLINICIAN-COLLECTED ANOGENITAL LESION SWAB SPECIMENS COMPARED WITH ELVIS ${ }^{\circledR}$ HSV ID AND D3 TYPING TEST AND SANGER SEQUENCING}

${ }^{1} S$ Young, ${ }^{2} \mathrm{~B}$ Van Der Pol, ${ }^{3} \mathrm{~S}$ Taylor, ${ }^{4} \mathrm{~K}$ Fife, ${ }^{2} \mathrm{E}$ Hook, ${ }^{5} \mathrm{R}$ Patel, ${ }^{6} \mathrm{~K}$ Ding, ${ }^{6} \mathrm{P}$ Hemyari, ${ }^{6}$ J Duncan, ${ }^{6} \mathrm{~S}$ Tang, ${ }^{6} \mathrm{O}$ Liesenfeld, ${ }^{6} \mathrm{~J}$ Osiecki ${ }^{*},{ }^{6} \mathrm{M}$ Lewinski. ${ }^{1}$ Tricore Reference Laboratories, Albuquerque, NM, USA; ${ }^{2}$ University of Alabama at Birmingham, Birmingham, AL, USA; ${ }^{3}$ Louisiana State University School of Medicine, New Orleans, LA, USA; ${ }^{4}$ ndiana University School of Medicine, Indianapolis, Indiana, USA; ${ }^{5}$ Royal South Hants Hospital, Southampton, England, UK; ${ }^{6}$ Roche Molecular Diagnostics, Pleasanton, CA, USA

\subsection{6/sextrans-2015-052270.339}

Introduction Diagnosis of anogenital herpes is definitively established by testing anogenital lesion specimens from symptomatic patients by culture or molecular methods. The objective of this study was to evaluate the cobas ${ }^{\circledR}$ HSV 1 and HSV 2 Test using clinician-collected swab specimens from external anogenital lesions as part of a large multicenter clinical trial conducted in the United States of America.

Methods Two swabs were collected from patients with possible HSV infection at 8 geographically diverse sites. The first swab was used for culture by the ELVIS ${ }^{\circledR}$ HSV ID and $\mathrm{D}^{3}$ Typing Test (Diagnostic Hybrids, Inc., Athens, OH) and PCR followed by Sanger sequencing for HSV-1 and HSV-2. The second swab was for the cobas ${ }^{\circledR}$ HSV 1 and 2 Test. Sensitivity and specificity were calculated compared to the combined results of culture and Sanger sequencing using the "any positive rule". The positive (PPA) and negative percent agreement (NPA) were calculated compared with culture.

Results There were 243 HSV positive subjects, with 84 HSV-1 (51 female, 33 male) and $167 \mathrm{HSV}-2$ (85 female, 82 male) positive subjects, among 408 evaluable participants (205 female, 203 male). The sensitivity and specificity of the cobas ${ }^{\circledR}$ HSV 1 and HSV 2 Test compared the Reference Method for HSV-1 was $92.9 \%(78 / 84)$ and $98.8 \%(320 / 324)$, respectively, and for HSV2 was $97.0 \%(162 / 167)$ and 94.6\% (228/241), respectively. The PPA and NPA of the cobas ${ }^{\circledR}$ HSV 1 and HSV 2 Test compared to the culture for HSV-1 was $100 \%(67 / 67)$ and $93.9 \%(199 /$ 212), respectively, and for HSV-2 was 99.2\% (128/129) and $83.2 \%(232 / 279)$, respectively.

Conclusion The cobas ${ }^{\circledR}$ HSV 1 and 2 Test displayed excellent performance compared to the combined results of culture and Sanger sequencing. The test is highly suitable to detect HSV in clinician-collected anogenital swab specimens from patients with suspected HSV infection.

Disclosure of interest statement This clinical trial study was supported by Roche Molecular Diagnostics.

\section{P07.24 PREDICTED INCLUSIVITY AND SPECIFICITY OF THE COBAS $^{\circledR} 4800$ CT/NG TEST THROUGH GLOBAL SURVEILLANCE MONITORING}

S Cayabyab Hibbard, X Chen, J Osiecki* ${ }^{*}$ C Honisch, M Lewinski, C Fillmore. Roche Molecular Diagnostics, Pleasanton, CA, USA

\section{$10.1136 /$ sextrans-2015-052270.340}

Introduction Nucleic acid amplification tests rely on conserved sequences for identification of specific targets, which may evolve, requiring global surveillance monitoring. An analysis of sequence heterogeneity within the primer and probe target region for the cobas ${ }^{\circledR} 4800 \mathrm{CT} / \mathrm{NG}$ Test was performed with publically available and in-house sequences to determine predicted inclusivity and specificity.

Methods To determine predicted inclusivity, analysis of the sequence heterogeneity within the primer and probe binding regions used in cobas ${ }^{\circledR} 4800 \mathrm{CT} / \mathrm{NG}$ Test were compared with the most current sequence information in NCBI's public sequence database supplemented with sequences generated by the Roche Global Surveillance Program. By design of the redundant nature of target amplification and detection for these assays (multiple copies of DR9 in NG and dual targets of ompA and cryptic plasmid in CT), mismatches in multiple sequences are required to affect assay inclusivity. For predicted specificity, the analysis of the potential generation of false-positive signals due to detection of non-target sequences was evaluated by interrogating the most current sequence information in NCBI's public sequence database.

Results For predicted inclusivity, a total of 56 cryptic plasmid and 373 ompA sequences from Chlamydia trachomatis and 357 sequences from 119 different strains of Neisseria gonorrhoeae covering the primer/probe binding region showed no predicted critical mismatches. For predicted specificity, extensive search identified no non- CT or NG target sequences that fit the broad criteria for potentially generating a false-positive signal based on the binding of two primers in the proper orientation, having a sequence that may bind one of the probes and generating a signal for an amplicon size of less than 3,000 base pairs.

Conclusion Global surveillance of publically available and inhouse generated sequences shows the cobas ${ }^{\circledR} 4800 \mathrm{CT} / \mathrm{NG}$ Test is a reliable molecular method for detection of Chlamydia trachomatis and Neisseria gonorrhoeae, displaying excellent predicted inclusivity and specificity.

Disclosure of interest The authors are employees of Roche Molecular Diagnostics which supported this study.

\section{P07.25 COMPARISON OF COBAS $®$ HSV $1 / 2$ TEST, QUIDEL LYRA $^{\text {TM }}$ DIRECT HSV 1+2/VZV, BD PROBETECTM HSV $1 / 2$ QX ASSAY AND SANGER SEQUENCING USING CLINICIAN-COLLECTED ANOGENITAL LESION SWABS}

${ }^{1} \mathrm{~S}$ Young, ${ }^{2} \mathrm{~B}$ Van Der Pol, ${ }^{3} \mathrm{~S}$ Taylor, ${ }^{4} \mathrm{~K}$ Fife, ${ }^{2} \mathrm{E}$ Hook, ${ }^{5} \mathrm{R}$ Patel, ${ }^{6} \mathrm{~K}$ Ding, ${ }^{6} \mathrm{P}$ Hemyari, ${ }^{6}$ J Duncan, ${ }^{6} \mathrm{~S}$ Tang, ${ }^{6} \mathrm{O}$ Liesenfeld, ${ }^{6} \mathrm{~J}$ Osiecki, ${ }^{6} \mathrm{M}$ Lewinski ${ }^{*}$. ${ }^{1}$ TriCore Reference Laboratories, Albuquerque, NM, USA; ${ }^{2}$ University of Alabama at Birmingham, Birmingham, AL, USA; ${ }^{3}$ Louisiana State University School of Medicine, New Orleans, LA, USA; ${ }^{4}$ Indiana University School of Medicine, Indianapolis, Indiana, USA; ${ }^{5}$ Royal South Hants Hospital, Southampton, England, UK; ${ }^{6}$ Roche Molecular Diagnostics, Pleasanton, CA, USA

10.1136/sextrans-2015-052270.341 